\title{
Patterns and Predictors of Trajectories of Depression after an Urban Disaster
}

\author{
ARIJIT NANDI, PHD, MELISSA TRACY, MPH, JOHN R. BEARD, MBBS, PHD, \\ DAVID VLAHOV, PHD, AND SANDRO GALEA, MD, DRPH
}

\begin{abstract}
PURPOSE: To identify and understand the patterns and predictors of depressive symptom trajectories over time after mass traumatic events.

METHODS: Data were used from a prospective, representative sample of adult residents of the New York City metropolitan area $(N=2,282)$ followed up across four survey waves between 2001 (after the September 11 attacks) and 2004. Semi-parametric group-based modeling was used to identify trajectories, as well as the time-fixed and time-varying predictors of distinct depressive trajectories.

RESULTS: Five distinct trajectories of depression were characterized: minimal symptomatology at all time points (group 1,39\% of sample), mild delayed depression (group 2, 34\% of sample), recovery (group $3,6 \%$ of sample), severe delayed depression (group 4, 13\% of sample), and chronic severe depression (group 5, $8 \%$ of sample). Among members of distinct trajectories, lower household income, exposure to ongoing stressors, and exposure to traumatic events were commonly associated with an increased number of depressive symptoms.

CONCLUSIONS: Ongoing socioeconomic adversity appears to be centrally associated with a worse course of depression after exposure to traumatic events. Identifying distinct trajectories of depression and the preventable factors that are associated with them may facilitate the development of interventions that aim to promote better mental health.

Ann Epidemiol 2009;19:761-770. (c) 2009 Elsevier Inc. All rights reserved.
\end{abstract}

KEY WORDS: Depression, Mental Disorders, Disasters.

\section{INTRODUCTION}

A growing body of epidemiologic research has assessed the burden of depressive psychopathology following exposure to disasters $(1-10)$, as well as in populations with increased exposure to traumatic events, including war veterans (11) and refugees (12-14). While comparisons across studies are complicated by substantial heterogeneity in the type of disaster, sample, and study design, cross-sectional research suggests that depressive symptomatology may be elevated in the immediate aftermath of a disaster, particularly among those with greater exposure to the event $(4,5,7,8)$. Results from prospective studies, in contrast, have been

From the Center for Population and Development Studies; Harvard School of Public Health; Boston, MA (A.N.); Department of Epidemiology, University of Michigan School of Public Health (M.T., S.G.) and Survey Research Center, Institute for Social Research (S.G.), Ann Arbor, MI; Center for Urban Epidemiologic Studies, New York Academy of Medicine (J.R.B., D.V., S.G.) and Department of Epidemiology, Columbia University Mailman School of Public Health (D.V., S.G.), New York, NY; School of Public Health, University of Sydney; Sydney (J.R.B.) and Faculty of Health and Applied Science, Southern Cross University, Lismore (J.R.B.), NSW, Australia.

Address correspondence to: Sandro Galea, MD, DrPH, Professor, Department of Epidemiology, University of Michigan, School of Public Health, 109 Observatory St, Room 3663, Ann Arbor, MI 48109-2029. Tel: (734) 647-9741. fax: (734) 763-5706. E-mail: sgalea@umich.edu.

Received June 2, 2009; accepted June 25, 2009. inconsistent, limiting inference regarding longitudinal patterns of depression after disasters. For example, some studies have observed a rapid resolution of depressive symptoms after exposure to mass trauma (1), whereas other work has shown a persistent burden with little evidence of recovery $(2,6)$.

Recent findings show that multiple subgroups may exist among those exposed to mass trauma, including those who are consistently free of symptoms, persistently affected, and with recurring symptomatology. For example, using a descriptive approach, we recently characterized patterns of depression over a 30-month period after the September 11, 2001 attacks in a population-based sample of New York City (NYC) metropolitan area residents. We found that $68 \%$ of participants did not meet criteria for depression in the 30 months after the September 11, 2001 attacks, whereas $30 \%$ experienced at least one episode of depression, and $2 \%$ were persistently depressed (15). These results are consistent with theoretical frameworks proposing that exposure to traumatic events may result in distinct trajectories of depressive symptoms ranging from an absence of symptoms to chronically severe levels of dysfunction (16).

A number of factors may predict membership in distinct trajectory subgroups following exposure to a disaster. Individuals with a history of traumatic event exposure or who have comorbid psychiatric conditions may experience greater 


\section{Selected Abbreviations and Acronyms}

BSI $=$ Brief Symptom Inventory

DSM-IV = Diagnostic and Statistical Manual of Mental Disorders, Fourth Edition.

$\mathrm{NYC}=$ New York City

SCID $=$ Structured Clinical Interview for Diagnostic and Statistical Manual of Mental Disorders, Third Edition Revised

depressive disruption over time (3). Peri-event characteristics, including a greater degree of exposure to a disaster event, may be associated with a more chronic course of psychopathology $(3,9,10)$. Additionally, the post-disaster environment may be important; ongoing exposure to stressful or traumatic events or, alternatively, having less access to social supports or other salutary resources, may predict membership in more symptomatic trajectories $(9,15)$.

There is growing evidence for heterogeneity in the longitudinal patterns of depression in population-based studies (17). However, analyses in populations exposed to mass traumatic events have generally treated depression as a homogenous outcome characterized by a common longitudinal course of symptoms and we are not aware of any work that has applied emerging statistical methods, such as latent growth modeling, to identify trajectories of depression in the aftermath of a disaster. In contrast to conventional growth modeling approaches, latent growth modeling approaches assign specific growth parameters to unobserved population subgroups with relatively homogeneous developmental paths $(18,19)$. This makes it possible to test whether distinct longitudinal trajectories of depression are present in a population and, relative to subjective classification strategies, avoids the identification of spurious clusters that may reflect random variation rather than true heterogeneity in longitudinal trajectories (20).

In this study, we extend our prior work (15) by using a latent class growth analysis to examine trajectories of depression, as well the time-fixed and time-varying determinants of distinct depressive trajectories, among a representative sample of adult residents of the NYC metropolitan area after the September 11, 2001 terrorist attacks.

\section{METHODS}

\section{Participants}

We conducted a random-digit-dial household survey to recruit baseline respondents approximately 6 months after the September 11 attacks (March 25-June 25, 2002). The sampling frame consisted of all adults (18 years of age and older) in the NYC metropolitan area. Interviews were conducted in English, Spanish, Mandarin, and Cantonese by trained interviewers using translated and back-translated questionnaires and a computer-assisted telephone interview system. Households were screened for eligibility by location. If eligible, an adult in each household was randomly selected by choosing the adult whose birthday was closest to the interview date. Up to 10 attempts were made to conduct the interview. Further details on this study are provided elsewhere (21).

Contact information was obtained for respondents, their key family members, and other important contacts. Three follow-up interviews were conducted approximately 6,18 , and 30 months after baseline (September 25, 2002-January 31, 2003; September 25, 2003-February 29, 2004; December 15, 2004-November 30, 2005). The mean interview time for all surveys was approximately 35 minutes. The Institutional Review Board at the New York Academy of Medicine approved the study and all study subjects provided oral consent at the time of the interview.

\section{Measures}

We used a modified version of the Structured Clinical Interview for Diagnostic and Statistical Manual of Mental Disorders, Third Edition Revised (SCID) (22), a validated approach that captures symptoms of major depression consistent with Diagnostic and Statistical Manual of Mental Disorders, Fourth Edition (DSM-IV) criteria (23), to assess symptoms of major depression. Respondents were asked about the presence for over 2 weeks of any of 10 symptoms of depression representing criterion A for major depression in DSM-IV. At the baseline interview, respondents who reported two or more symptoms that occurred together in a 1-month period were asked about the timing of their most recent symptoms; symptoms were categorized as having occurred before or since the September 11 attacks. The timing of symptoms for respondents who reported either one symptom of depression or two or more symptoms of depression that did not occur together in a 1 -month period was unavailable, and these symptoms were categorized as having occurred before the September 11, 2001 attacks. Consistent with DSM-IV guidelines, lifetime depression at baseline was defined as having five or more symptoms for at least 2 weeks at any time in the past. At subsequent follow-up interviews, we asked about the number of symptoms experienced for at least 2 weeks since the prior interview.

The Cronbach alpha for 10 items used in our depression scale was 0.79 (24). Furthermore, in a prior validation study comparing our instrument, with depression defined dichotomously as five symptoms experienced for at least 2 weeks, with the Brief Symptom Inventory (BSI) (25), the BSI depression scale had a sensitivity of $73 \%$ and specificity of $87 \%$ as classified by our instrument (26). In addition to being used in previous surveys focusing on the effects of the September 11 attacks, this scale has also been used in other population surveys $(5,27)$. 
Time-fixed covariates were assessed at baseline. We asked about sociodemographic factors, including age, gender, race/ethnicity, marital status, and educational attainment. To evaluate pre-event stressors, we asked respondents if they experienced divorce or separation, marriage, family problems, problems at work, or unemployment in their lifetime prior to the September 11 attacks. We asked about exposure to any of 12 traumatic events (e.g., natural disaster; accident at work, in a car, or somewhere else; assault with a weapon; unwanted sexual contact; death of a spouse or mate) in the time prior to the September 11 attacks. To assess whether respondents were directly affected by the September 11 attacks, we asked if they were in the World Trade Center on September 11, injured during the attacks, had a friend or relative killed, had possessions lost or damaged, lost a job because of the attacks, or were involved in the rescue effort. In the analysis, exposure to pre-event stressors, exposure to pre-event traumatic events, and direct exposure to the September 11 attacks were dichotomized. To evaluate social support we asked about emotional (e.g., "someone to love you and make you feel wanted"), instrumental (e.g., "someone to help you if you were confined to bed"), and appraisal (e.g., "someone to give you good advice in a crisis") support in the 6 months prior to the September 11 attacks using an abbreviated version of the Medical Outcomes Study social support scale (28). The Cronbach alpha for the five-item modified version of this scale was 0.90 (29). For the analysis, social support scores were summed and categorized as low, medium, or high based on the tertiles of social support reported in the sample. To assess peri-event emotional reactions to the September 11 attacks, we used a modified version of the Diagnostic Interview Schedule subscale for panic attack (30), phrased to detect symptoms experienced "in the first few hours" after the September 11 terrorist attacks. Respondents who reported at least four out of a total of 16 symptoms were classified as having had a peri-event emotional reaction. Further information on the measurement of peri-event emotional reactions is provided elsewhere (31).

Time-varying covariates, including household income, stressors, and traumatic events, were assessed at each interview. Ongoing stressors and traumatic events were measured using the same items as time-fixed stressors and traumatic events, as described above. At baseline, respondents reported their experience of stressors and traumatic events since the September 11 attacks; in follow-up interviews, respondents reported their experience of stressors and traumatic events since the prior interview. Time-varying variables for ongoing stressful and traumatic events were dichotomized for analysis into those who did or did not experience any stressors or traumatic events at each time point. Household income was updated at each interview and was dichotomized as less than $\$ 30,000$ or greater than or equal to $\$ 30,000$ for this analysis.

\section{Statistical Analyses}

Sampling weights were developed and applied to the data to correct for potential selection bias relating to the number of household telephones, persons in the household, and over-sampling. We also applied post-stratification weights to make the follow-up survey samples demographically representative of the NYC metropolitan area population according to the 2000 U.S. census (32).

We used semi-parametric group-based modeling, a type of latent growth curve analysis, to identify trajectories of depressive symptoms across survey waves and to assess determinants of trajectory group membership $(20,33)$. We fit zero-inflated Poisson regression models to account for the greater number of zeros in the count of depressive symptoms than would be expected under the Poisson distribution. The Bayesian Information Criterion was used to select the bestfitting model. After selecting the model with the optimal number of trajectory groups, we determined the appropriate shape of each trajectory group (i.e., linear, quadratic, cubic) based on statistical significance $(p<0.05)$. For each individual, we calculated the probability of belonging to each trajectory group. Individuals were then assigned to the group with the largest posterior probability. Adjusted analyses were conducted by contemporaneously adding time-fixed and time-varying covariates to the best-fitting trajectory models; time-fixed covariates were added assuming they affected the probability of group membership and timevarying covariates were added assuming they affected the expected number of depressive symptoms within each trajectory group. Only variables that were significantly associated with trajectory group membership in bivariate models were considered in adjusted models.

\section{RESULTS}

\section{Sample Characteristics}

Of the 2,752 total baseline participants, 2,282 completed at least one follow-up interview and were included in the study sample. The respondents included were comparable to both the total baseline sample and the general population of the NYC metropolitan area with respect to basic demographic characteristics (15). The distributions of time-fixed and time-varying covariates for the baseline and three followup waves are shown in Table 1 . At baseline, the mean age was 44.7 years, $45.2 \%$ of respondents were male, $60.0 \%$ were married, $64.5 \%$ had at least some college education, and the racial/ethnic composition was $55.5 \%$ white, $5.4 \%$ Asian, $16.6 \%$ black, $18.5 \%$ Hispanic, and $4.1 \%$ other race/ethnicity. Of baseline respondents, $20.6 \%$ reported at 
TABLE 1. Distributions of time-fixed covariates, time-varying covariates, and depressive symptoms across study waves among residents of the NYC metropolitan area aft the September 11, 2001 attacks $(N=2,282)$

\begin{tabular}{|c|c|c|c|c|c|c|c|c|}
\hline & \multicolumn{2}{|c|}{ Wave 1} & \multicolumn{2}{|c|}{ Wave 2} & \multicolumn{2}{|c|}{ Wave 3} & \multicolumn{2}{|c|}{ Wave 4} \\
\hline & No. & Weighted \% & No. & Weighted \% & No. & Weighted \% & No. & Weighted \% \\
\hline & \multicolumn{8}{|c|}{ Time-fixed covariates } \\
\hline Total & 2282 & & 1939 & & 1832 & & 1610 & \\
\hline \multicolumn{9}{|l|}{ Age } \\
\hline $18-24$ & 202 & 12.98 & 164 & 13.72 & 142 & 13.64 & 128 & 13.72 \\
\hline $25-34$ & 503 & 21.42 & 389 & 23.90 & 356 & 23.89 & 311 & 23.84 \\
\hline $35-44$ & 499 & 20.97 & 424 & 20.61 & 420 & 20.63 & 339 & 20.81 \\
\hline $45-54$ & 450 & 19.76 & 398 & 18.96 & 377 & 19.00 & 343 & 19.01 \\
\hline $55-64$ & 299 & 13.24 & 266 & 12.24 & 252 & 12.23 & 237 & 12.20 \\
\hline$\geqslant 65$ & 307 & 11.63 & 277 & 10.57 & 269 & 10.61 & 236 & 10.63 \\
\hline \multicolumn{9}{|l|}{ Gender } \\
\hline Male & 1034 & 45.19 & 869 & 45.75 & 807 & 45.71 & 723 & 45.74 \\
\hline Female & 1248 & 54.81 & 1070 & 54.25 & 1025 & 54.29 & 887 & 54.26 \\
\hline \multicolumn{9}{|l|}{ Race/ethnicity } \\
\hline White & 1363 & 55.50 & 1188 & 53.11 & 1130 & 53.18 & 1017 & 53.15 \\
\hline Asian & 129 & 5.35 & 94 & 5.38 & 100 & 5.36 & 80 & 5.36 \\
\hline Black & 317 & 16.60 & 265 & 16.73 & 248 & 16.68 & 209 & 16.69 \\
\hline Latino & 364 & 18.49 & 293 & 20.63 & 269 & 20.62 & 226 & 20.63 \\
\hline Other & 75 & 4.06 & 67 & 4.16 & 58 & 4.17 & 53 & 4.16 \\
\hline \multicolumn{9}{|l|}{ Marital status } \\
\hline Married & 986 & 50.95 & 845 & 49.72 & 821 & 51.72 & 719 & 51.31 \\
\hline Divorced/Separated/Widowed & 471 & 16.30 & 411 & 15.53 & 389 & 14.54 & 334 & 14.06 \\
\hline Single/Unmarried couple & 816 & 32.75 & 675 & 34.75 & 614 & 33.73 & 553 & 34.63 \\
\hline \multicolumn{9}{|l|}{ Education } \\
\hline Graduate work/College degree & 1139 & 42.48 & 987 & 43.16 & 930 & 43.48 & 837 & 44.20 \\
\hline Some college & 434 & 21.99 & 364 & 21.67 & 357 & 22.48 & 315 & 23.03 \\
\hline High school & 481 & 25.57 & 405 & 24.97 & 385 & 25.38 & 328 & 24.18 \\
\hline Less than high school & 219 & 9.96 & 176 & 10.19 & 156 & 8.66 & 123 & 8.59 \\
\hline \multicolumn{9}{|l|}{ Pre-September 11 stressors } \\
\hline 0 & 1785 & 79.43 & 1527 & 79.91 & 1444 & 79.79 & 1281 & 80.28 \\
\hline$\geqslant 1$ & 497 & 20.57 & 412 & 20.09 & 388 & 20.21 & 329 & 19.72 \\
\hline \multicolumn{9}{|l|}{ Pre-September 11 traumatic events } \\
\hline 0 & 530 & 23.23 & 425 & 22.68 & 423 & 23.68 & 372 & 23.73 \\
\hline $1-2$ & 990 & 45.08 & 857 & 44.90 & 803 & 45.84 & 694 & 45.64 \\
\hline$\geqslant 3$ & 762 & 31.69 & 657 & 32.42 & 606 & 30.48 & 544 & 30.63 \\
\hline \multicolumn{9}{|l|}{ Directly affected by September $11^{\mathrm{d}}$} \\
\hline No & 1622 & 71.96 & 1383 & 71.93 & 1294 & 71.65 & 1137 & 70.86 \\
\hline Yes & 660 & 28.04 & 556 & 28.07 & 538 & 28.35 & 473 & 29.14 \\
\hline \multicolumn{9}{|l|}{ Social support } \\
\hline High & 882 & 39.12 & 756 & 39.29 & 700 & 39.08 & 638 & 40.65 \\
\hline Medium & 663 & 31.12 & 565 & 31.24 & 542 & 30.85 & 470 & 30.38 \\
\hline Low & 702 & 29.76 & 589 & 29.47 & 561 & 30.07 & 480 & 28.97 \\
\hline \multicolumn{9}{|l|}{ Peri-event emotional reaction } \\
\hline No & 1949 & 86.29 & 1657 & 86.71 & 1564 & 86.30 & 1380 & 86.07 \\
\hline Yes & 339 & 13.71 & 282 & 13.29 & 268 & 13.70 & 230 & 13.93 \\
\hline Lifetime depression & & & & & & & & \\
\hline No & & & & & & & & \\
\hline Yes & 543 & 19.92 & 467 & 20.40 & 445 & 19.71 & 405 & 21.34 \\
\hline & & & & Time-vary & ng covariates & & & \\
\hline Low household income & & & & & & & & \\
\hline$\geqslant \$ 30,000$ & 1368 & 72.09 & 1131 & 68.55 & 1119 & 71.72 & 1027 & 71.35 \\
\hline$<\$ 30,000$ & 537 & 27.91 & 504 & 31.45 & 474 & 28.28 & 418 & 28.65 \\
\hline Ongoing stressors & & & & & & & & \\
\hline 0 & 2054 & 90.95 & 1337 & 69.26 & 1227 & 66.55 & 1053 & 66.04 \\
\hline$\geqslant 1$ & 228 & 9.05 & 602 & 30.74 & 605 & 33.45 & 557 & 33.96 \\
\hline Ongoing traumas & & & & & & & & \\
\hline 0 & 1956 & 86.14 & 1330 & 67.30 & 1025 & 55.25 & 846 & 54.00 \\
\hline$\geqslant 1$ & 326 & 13.86 & 609 & 32.70 & 807 & 44.75 & 764 & 46.00 \\
\hline Depressive symptoms, mean (SE) & $0.82(2.22)$ & & $1.85(2.58)$ & & $1.98(2.67)$ & & $2.11(2.78)$ & \\
\hline
\end{tabular}

$\mathrm{NYC}=$ New York City; SE $=$ standard error (of the mean). 
least one pre-September 11 th stressor and 9.1\% experienced at least one ongoing stressor between the September 11th attacks and their baseline interview. At least one preSeptember traumatic event and ongoing traumatic event were reported by $76.8 \%$ and $13.9 \%$ of respondents, respectively. Twenty-eight percent of respondents were directly affected by the attacks and $13.7 \%$ experienced a peri-event emotional reaction. At baseline, $27.9 \%$ of respondents reported less than $\$ 30,000$ in household income. The mean numbers of depressive symptoms were $0.82,1.85,1.98$, and 2.11 at waves $1,2,3$, and 4 , respectively.

\section{Trajectories of Depressive Symptoms}

Using the Bayesian information criterion to compare models with different numbers of trajectory groups, we found that a model with five groups provided the best fit for the patterns of depressive symptoms in our sample. As shown in Table 2, the depressive symptom growth curves for groups 1 and 5 were best defined by intercept, linear, and quadratic terms, whereas parameter estimates for groups 2,3 , and 4 suggested cubic trends. The average posterior probabilities of group assignment ranged from 0.733 to 0.924 for the trajectory groups. Growth curves for the five groups are shown in Fig. 1. Three groups were described by the absence of depressive symptomatology at baseline, but were distinguished by distinct increases in the number of symptoms over the study period. On average, respondents in group 1 (39.4\% of the sample) gradually increased to fewer than one symptom of depression over the course of follow-up. In the 6 months after baseline, respondents in groups 2 and 4 showed average increases of 1.5 and 5 depressive symptoms, respectively. Thereafter, respondents in group 2 (33.5\% of the sample) increased to two symptoms at the end of the study period, whereas the number of depressive symptoms among respondents in group 4 (13.2\% of the sample) increased by approximately one symptom between 6 and 18 months after baseline and then decreased gradually until the end of the study period. Two groups were characterized by depressive symptomatology at baseline. On average, respondents in group 3 (5.6\% of the sample) showed a decrease from approximately five depressive symptoms to one symptom in the 6 months after baseline, followed by a gradual decline in symptomatology until the end of the study period. Group 5 (8.3\% of the sample) declined from approximately seven to five and a half depressive symptoms in the first 6 months after baseline and then showed an average increase to six symptoms at the end of the study period.

\section{Determinants of Depressive Trajectory Groups}

The results of a fully adjusted model assessing the relation between time-fixed and time-varying covariates and membership in depressive symptom trajectory groups are

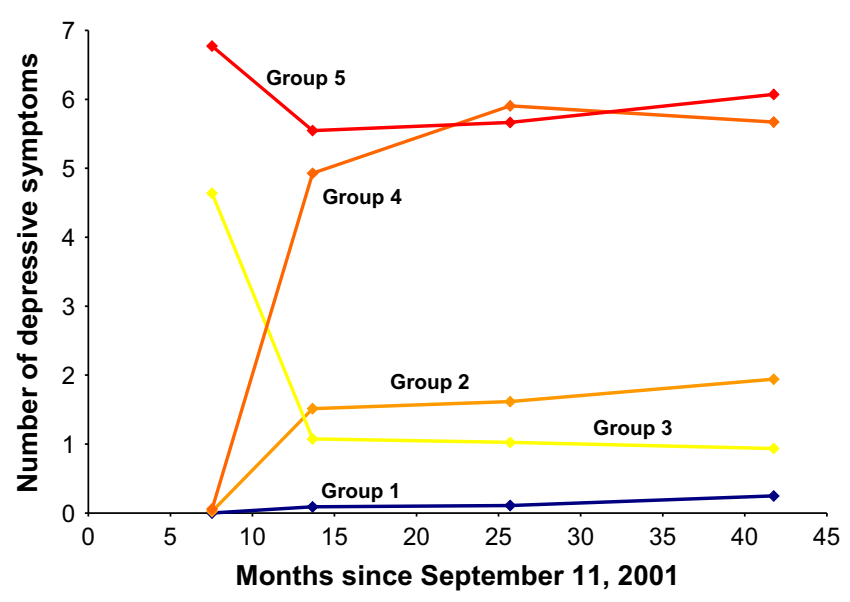

FIGURE 1. Trajectories of depressive symptoms among residents of the NYC metropolitan area after the September 11, 2001 attacks $(N=2,282)$.

presented in Table 3. Women were more likely than men to have been assigned to group 4 relative to group 1 , the group that showed minimal symptomatology over time. Black or Latino race/ethnicity, compared to white, was associated with membership in groups 2,4 , and 5 relative to group 1. Relative to married respondents, single respondents were more likely to be in groups 2, 4, and 5 relative to group 1 , whereas divorced, separated, or widowed respondents were less likely to be in group 3 relative to group 1 . Relative to respondents with at least a college degree, those with lower educational attainment were more likely to be assigned to groups 2, 4, and 5 and less likely to be assigned to the group 3 compared to group 1. Exposure to at least one pre-September 11 stressor compared to none was associated with membership in groups 4 and 5 compared to group 1. Exposure to at least three pre-September 11 traumatic events compared to none was associated with membership in groups 3 and 4 relative to the group 1 . Those who were directly affected by the September 11th attacks were more likely than those who were not to be assigned to group 3 relative to the group 1. Participants with low to medium levels of social support compared to higher levels were more likely to be assigned to groups 4 and 5 relative to group 1 . Those who experienced a peri-event emotional reaction were more likely than those who did not to be in group 5 relative to group 1. Those who reported lifetime depression prior to the attacks were more likely than those who did not to be assigned to groups 3, 4, and 5 relative to group 1 .

Among time-varying covariates, we found that lower household income relative to higher income was associated with an increased number of depressive symptoms for members of group 4. Reporting one or more ongoing stressors relative to none was associated with an increased number of depressive symptoms for members of groups 
TABLE 2. Parameter estimates, prevalence, and mean posterior probability of assignment for each trajectory group of depressive symptoms among residents of the NYC metropolitan area after the September 11, 2001 attacks $(2,282)$

\begin{tabular}{|c|c|c|c|c|c|}
\hline & Parameter & Estimate (SE) & $p$ Value & Mean posterior probability (SD) & Prevalence \\
\hline \multirow[t]{3}{*}{ Group 1} & Intercept & $-5.084(0.570)$ & $<0.001$ & $0.733(0.242)$ & 39.4 \\
\hline & Linear & $0.160(0.036)$ & $<0.001$ & - & - \\
\hline & Quadratic & $-0.002(0.001)$ & 0.003 & - & - \\
\hline \multirow[t]{4}{*}{ Group 2} & Intercept & $-7.302(0.657)$ & $<0.001$ & $0.889(0.135)$ & 33.5 \\
\hline & Linear & $0.964(0.102)$ & $<0.001$ & - & - \\
\hline & Quadratic & $-0.036(0.004)$ & $<0.001$ & - & - \\
\hline & Cubic & $0.0004(0.0001)$ & $<0.001$ & - & - \\
\hline \multirow[t]{4}{*}{ Group 3} & Intercept & $4.772(0.753)$ & $<0.001$ & $0.846(0.196)$ & 5.6 \\
\hline & Linear & $-0.597(0.152)$ & $<0.001$ & - & - \\
\hline & Quadratic & $0.023(0.007)$ & 0.002 & - & - \\
\hline & Cubic & $-0.0003(0.0001)$ & 0.006 & - & - \\
\hline \multirow[t]{4}{*}{ Group 4} & Intercept & $-7.216(0.478)$ & $<0.001$ & $0.924(0.124)$ & 13.2 \\
\hline & Linear & $1.108(0.077)$ & $<0.001$ & - & - \\
\hline & Quadratic & $-0.042(0.003)$ & $<0.001$ & - & - \\
\hline & Cubic & $0.0005(0.0001)$ & $<0.001$ & - & - \\
\hline \multirow[t]{3}{*}{ Group 5} & Intercept & $2.113(0.073)$ & $<0.001$ & $0.866(0.163)$ & 8.3 \\
\hline & Linear & $-0.036(0.011)$ & 0.001 & - & - \\
\hline & Quadratic & $0.001(0.0002)$ & 0.003 & - & - \\
\hline
\end{tabular}

$\mathrm{SE}=$ standard error; $\mathrm{SD}=$ standard deviation

2 and 4. Finally, reporting exposure to one or more ongoing traumatic events compared to none was associated with an increased number of depressive symptoms for those assigned to groups $1,2,4$, and 5 .

\section{DISCUSSION}

\section{Trajectories of Depressive Symptoms}

Our findings suggest that the epidemiology of depression after exposure to mass trauma is more adequately described by a heterogeneous set of distinct trajectory subgroups than a homogenous population-average level of symptoms over time. Using the DSM-IV, which defines depression based on the presence of five or more symptoms experienced during at least a 2 -week period, as a rough guideline we can categorize distinct trajectories as clinically or not clinically relevant. We found that the most common trajectory of depression after the September 11 attacks was group 1, which was characterized by resistance, or negligible levels of depressive symptoms over time; in contrast, fewer than $10 \%$ of respondents were assigned to group 5, characterized by chronic levels of severe symptomatology. These findings corroborate the prevailing observation that most individuals exposed to mass trauma maintain a relatively stable trajectory of healthy functioning, whereas a smaller proportion are persistently depressed (15). We identified three inconstant trajectories that showed changes in depressive severity over time. Almost $50 \%$ of respondents were assigned to groups 2 and 4, which showed an increase in symptomatology that persisted at mild and severe levels, respectively. Consistent with other trauma research (11), these results suggest that delayed depression may be a common trajectory of depression following disasters. Members of group 3 showed a monotonic decline in depressive symptoms over time that is characteristic of research assessing the longitudinal course of depression in samples anchored to a specific event, be they clinical samples or samples collected after mass traumas (27). This group was characterized by clinically relevant levels of depression at baseline; however, by the end of follow-up, levels of depressive symptoms in this group approached mild levels, suggesting that this trajectory represented recovery rather than resilience, which is described in the literature by a more rapid return to normal functioning (16).

\section{Determinants of Depressive Trajectory Groups}

Our findings suggest that trajectory groups are distinguished by a number of factors. Sociodemographic characteristics, including race/ethnicity and marital status, frequently discriminated between the trajectory groups, even after adjusting for other factors including exposure to stressors, exposure to traumatic events, and social support. For example, relative to whites, we found that blacks and Latinos were more likely to be assigned to the three trajectory groups characterized by persistent levels of depressive symptoms than the resistance group. Similarly, single compared to married status and less compared to more educational attainment were both associated with membership in groups characterized by persistent depressive symptomatology. There are a number of potential explanations for these important sociodemographic differences between groups. For example, differential access to salutary resources 
TABLE 3. Adjusted model assessing the relation between time-fixed and time-varying covariates and trajectory group membership among NYC metropolitan area residents after the September 11, 2001 attacks $(N=2,282)$

\begin{tabular}{|c|c|c|c|c|c|c|c|c|c|c|}
\hline & \multicolumn{2}{|c|}{ Group 1} & \multicolumn{2}{|c|}{ Group 2} & \multicolumn{2}{|c|}{ Group 3} & \multicolumn{2}{|c|}{ Group 4} & \multicolumn{2}{|c|}{ Group 5} \\
\hline & Estimate (SE) & $p$ Value* & Estimate (SE) & $p$ Value & Estimate (SE) & $p$ Value & Estimate (SE) & $p$ Value & Estimate (SE) & $p$ Value \\
\hline & \multicolumn{10}{|c|}{ Time-fixed covariates $^{\dagger}$} \\
\hline \multicolumn{11}{|l|}{ Age } \\
\hline $18-24$ & 0.000 & - & 0.000 & - & 0.000 & - & 0.000 & - & 0.000 & - \\
\hline $25-34$ & 0.000 & - & $0.246(0.649)$ & 0.704 & $0.150(0.558)$ & 0.788 & $0.741(0.572)$ & 0.195 & $-0.390(0.718)$ & 0.587 \\
\hline $35-44$ & 0.000 & - & $0.620(1.017)$ & 0.542 & $-0.068(0.659)$ & 0.918 & $1.286(0.626)$ & 0.040 & $0.268(0.748)$ & 0.720 \\
\hline $45-54$ & 0.000 & - & $0.484(0.945)$ & 0.609 & $-0.119(0.657)$ & 0.857 & $1.064(0.625)$ & 0.089 & $-0.100(0.753)$ & 0.894 \\
\hline $55-64$ & 0.000 & - & $0.534(0.892)$ & 0.550 & $-0.257(0.869)$ & 0.768 & $0.540(0.628)$ & 0.390 & $-0.657(0.846)$ & 0.438 \\
\hline$\geqslant 65$ & 0.000 & - & $1.650(0.872)$ & 0.058 & $-0.423(1.068)$ & 0.692 & $1.940(0.669)$ & 0.004 & $-1.038(1.135)$ & 0.360 \\
\hline \multicolumn{11}{|l|}{ Gender } \\
\hline Male & 0.000 & - & 0.000 & - & 0.000 & - & 0.000 & - & 0.000 & - \\
\hline Female & 0.000 & - & $0.410(0.412)$ & 0.320 & $0.531(0.366)$ & 0.146 & $1.043(0.279)$ & $<0.001$ & $0.724(0.423)$ & 0.087 \\
\hline \multicolumn{11}{|l|}{ Race/ethnicity } \\
\hline White & 0.000 & - & 0.000 & - & 0.000 & - & 0.000 & - & 0.000 & - \\
\hline Asian & 0.000 & - & $0.023(0.900)$ & 0.980 & $-0.196(0.676)$ & 0.772 & $0.092(0.504)$ & 0.854 & $-0.587(1.184)$ & 0.620 \\
\hline Black & 0.000 & - & $1.225(0.493)$ & 0.013 & $0.440(0.852)$ & 0.605 & $1.156(0.429)$ & 0.007 & $1.186(0.552)$ & 0.032 \\
\hline Latino & 0.000 & - & $1.314(0.531)$ & 0.013 & $0.679(0.719)$ & 0.345 & $0.932(0.468)$ & 0.047 & $1.105(0.598)$ & 0.065 \\
\hline Other & 0.000 & - & $-0.812(1.117)$ & 0.467 & $-1.079(1.447)$ & 0.456 & $-0.171(0.546)$ & 0.754 & $-1.166(0.850)$ & 0.170 \\
\hline \multicolumn{11}{|l|}{ Marital status } \\
\hline Married & 0.000 & - & 0.000 & - & 0.000 & - & 0.000 & - & 0.000 & - \\
\hline Divorced/Separated/Widowed & 0.000 & - & $-0.610(0.607)$ & 0.315 & $-1.404(0.698)$ & 0.044 & $0.069(0.348)$ & 0.843 & $0.684(0.537)$ & 0.203 \\
\hline Single/Unmarried couple & 0.000 & - & $1.196(0.587)$ & 0.042 & $0.618(0.478)$ & 0.196 & $1.117(0.378)$ & 0.003 & $1.924(0.503)$ & $<0.001$ \\
\hline \multicolumn{11}{|l|}{ Education } \\
\hline Graduate work/College degree & 0.000 & - & 0.000 & - & 0.000 & - & 0.000 & - & 0.000 & - \\
\hline Some college & 0.000 & _- & $0.142(0.420)$ & 0.735 & $-0.794(0.564)$ & 0.159 & $0.016(0.326)$ & 0.961 & $0.695(0.458)$ & 0.129 \\
\hline High school & 0.000 & - & $1.011(0.417)$ & 0.015 & $0.288(0.546)$ & 0.598 & $0.973(0.342)$ & 0.004 & $1.740(0.522)$ & 0.001 \\
\hline Less than high school & 0.000 & - & $-0.521(1.208)$ & 0.667 & $-15.593(1.155)$ & $<0.001$ & $0.103(0.774)$ & 0.894 & $1.228(0.884)$ & 0.165 \\
\hline \multicolumn{11}{|l|}{ Pre-September 11 stressors } \\
\hline 0 & 0.000 & - & 0.000 & - & 0.000 & - & 0.000 & - & 0.000 & - \\
\hline$\geqslant 1$ & 0.000 & - & $0.978(0.545)$ & 0.073 & $0.764(0.496)$ & 0.124 & $1.317(0.378)$ & 0.001 & $2.056(0.476)$ & $<0.001$ \\
\hline \multicolumn{11}{|c|}{ Pre-September 11 traumatic events } \\
\hline 0 & 0.000 & - & 0.000 & - & 0.000 & - & 0.000 & - & 0.000 & - \\
\hline $1-2$ & 0.000 & - & $0.449(0.567)$ & 0.429 & $-0.175(0.583)$ & 0.765 & $0.778(0.343)$ & 0.023 & $0.267(0.578)$ & 0.644 \\
\hline$\geqslant 3$ & 0.000 & _- & 0.545 & 0.386 & $1.101(0.527)$ & 0.037 & $0.932(0.373)$ & 0.013 & $-0.023(0.588)$ & 0.969 \\
\hline \multicolumn{11}{|c|}{ Directly affected by September $11^{\ddagger}$} \\
\hline No & 0.000 & - & 0.000 & - & 0.000 & - & 0.000 & - & 0.000 & - \\
\hline Yes & 0.000 & - & $-0.075(0.448)$ & 0.868 & $0.909(0.450)$ & 0.043 & $0.037(0.319)$ & 0.907 & $0.471(0.432)$ & 0.276 \\
\hline \multicolumn{11}{|l|}{ Social support } \\
\hline High & 0.000 & - & 0.000 & - & 0.000 & - & 0.000 & - & 0.000 & - \\
\hline Medium & 0.000 & - & $0.727(0.485)$ & 0.134 & $-0.389(0.443)$ & 0.380 & $0.696(0.302)$ & 0.021 & $0.999(0.493)$ & 0.043 \\
\hline Low & 0.000 & - & $1.163(0.468)$ & 0.013 & $-0.773(0.835)$ & 0.355 & $1.381(0.346)$ & $<0.001$ & $2.092(0.451)$ & $<0.001$ \\
\hline \multicolumn{11}{|l|}{ Peri-event emotional reaction } \\
\hline No & 0.000 & - & 0.000 & - & 0.000 & - & 0.000 & - & 0.000 & - \\
\hline Yes & 0.000 & - & $1.134(0.656)$ & 0.084 & $0.865(0.664)$ & 0.193 & $0.767(0.533)$ & 0.150 & $1.806(0.573)$ & 0.002 \\
\hline
\end{tabular}




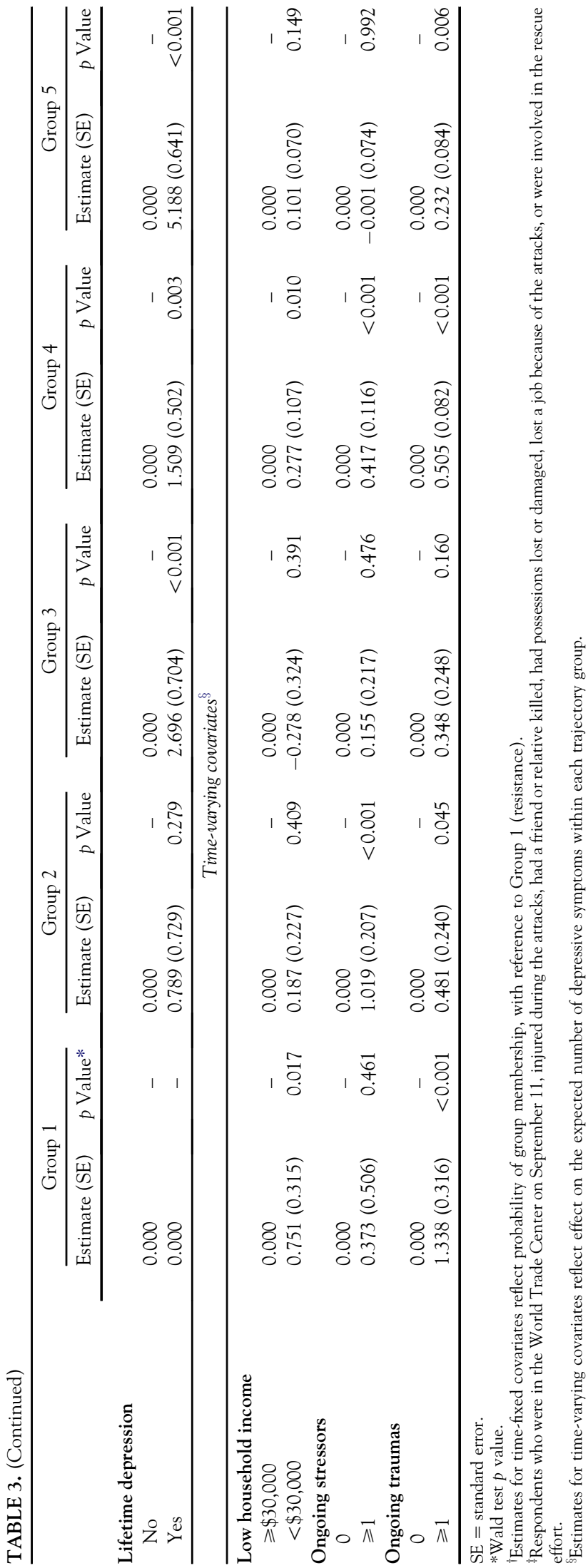

not considered in this study, including access to mental health care and stable employment, may explain differences by race/ethnicity, marital status, and education (34-36).

Respondents with greater exposure to pre-event stressors and traumatic events and fewer social supports to buffer them were more likely to be assigned to highly symptomatic versus less symptomatic depressive groups. For example, we found that the two groups with severe levels of symptoms, the chronic severe depression and severe delayed depression groups, were the only groups that were more likely to report exposure to pre-event stressors relative to the resistance group. Exposure to prior stressors may exacerbate levels of depression in the aftermath of a disaster. Furthermore, we found that respondents who experienced a peri-event emotional reaction in the first few hours after the September 11th attacks were more likely than those who did not to be assigned to the group with chronic severe depression, relative to the resistance group. Although the temporality is indeterminate, a peri-event emotional reaction may be an important predictor of persistent psychopathology following traumatic event experiences. Finally, those reporting a lifetime history of depression were more likely than respondents who did not to be assigned to groups with a higher initial number of depressive symptoms.

Among time-varying covariates, we found that lower household income and exposure to ongoing stressors and traumatic events were associated with an increased number of depressive symptoms in various trajectory groups, with the exception of the recovery group. Our findings corroborate recent work showing that decreased standards of material living were associated with increased depressive symptomatology in a general population sample (37). Post-migration stressors have also been associated with increased depression scores in a sample of refugee children (12). In disaster settings, our findings suggest that the experience of ongoing adversity may function to protract levels of depression over time. These results highlight the importance of considering ongoing stressful circumstances, as well as the material resources for adequately coping with them, to improve trajectories of depression after exposure to mass trauma.

\section{Limitations}

There are several limitations to this study. First, we used telephone interviews to assess depressive symptoms. Although it has been shown that telephone and in-person assessment of Axis I disorders, including depression, result in comparable estimates of symptomatology $(38,39)$, depressive symptoms collected via self-report cannot be equated to depression assessed by a trained clinician. Second, our sampling frame excluded respondents without a household telephone. However, we found that there 
were no significant differences in the distribution of key demographic characteristics between the sample included in this analysis and the general population of the NYC metropolitan area, both at baseline and during subsequent follow-up waves (32). Third, it is possible that losses to follow-up may have biased our sample. However, we found that the application of censoring weights in another study using the same data set did not appreciably alter results (15), suggesting that our findings are not sensitive to the degree of loss to follow-up that occurred in our sample. Fourth, we were unable to control for medication use. However, we did adjust for lifetime depression, which was associated with membership in more symptomatic groups compared to the resistance group, suggesting that assignment to the resistance group was not an artifact of antidepressive medication use. Fifth, in order to achieve the convergence of our statistical models, we dichotomized time-varying covariates. This may have introduced measurement error. Finally, because of data limitations, the timing of depressive symptoms was categorized as having occurred before the September 11, 2001 attacks for respondents who reported one symptom or two or more symptoms that did not occur together in a 1-month period. In separate analyses we assessed the sensitivity of our results to this assumption and found few qualitative differences in terms of either the patterns or predictors of depressive symptom trajectories in our sample.

\section{CONCLUSIONS}

We found evidence of distinct trajectories of symptoms of depression in a representative sample of adult residents of the NYC metropolitan area after the September 11 attacks. Our results suggest that the number of depressive symptoms endorsed by an individual following exposure to mass trauma may not be an appropriate prognosticator for targeted intervention. For example, at any particular occasion, cross-sectional analyses imply that individuals with the same number of depressive symptoms are categorically similar. However, our analyses suggest that individuals with the same number of depressive symptoms at any particular time may belong to clinically distinct subgroups. This underlines the importance of identifying distinct depressive trajectories rather than assuming that all persons follow a similar clinical course.

We also found that the determinants of depressive symptoms vary by trajectory group. Our analysis of the time-fixed and time-varying covariates suggested that factors including race/ethnicity, education, income, and exposure to stressors and trauma might underlie the gradient we observed in susceptibility to depression. Future work aimed at building models to predict depressive trajectory membership among individuals exposed to trauma may facilitate targeted interventions that reduce the burden of depression after disasters.

This research was supported in part by grants MH 066391, DA 022720, and $\mathrm{MH} 082729$.

\section{REFERENCES}

1. North CS, Kawasaki A, Spitznagel EL, Hong BA. The course of PTSD, major depression, substance abuse, and somatization after a natural disaster. J Nerv Ment Dis. 2004;192:823-829.

2. Fullerton CS, Ursano RJ, Wang L. Acute stress disorder, posttraumatic stress disorder, and depression in disaster or rescue workers. Am J Psychiatry. 2004;161:1370-1376

3. Nolen-Hoeksema S, Morrow J. A prospective study of depression and posttraumatic stress symptoms after a natural disaster: the 1989 Loma Prieta Earthquake. J Pers Soc Psychol. 1991;61:115-121.

4. Person C, Tracy M, Galea S. Risk factors for depression after a disaster. J Nerv Ment Dis. 2006;194:659-666.

5. Galea S, Ahern J, Resnick H, Kilpatrick D, Bucuvalas M, Gold J, et al. Psychological sequelae of the September 11 terrorist attacks in New York City. N Engl J Med. 2002;346:982-987.

6. Goenjian AK, Walling D, Steinberg AM, Karayan I, Najarian LM, Pynoos R. A prospective study of posttraumatic stress and depressive reactions among treated and untreated adolescents 5 years after a catastrophic disaster. Am J Psychiatry. 2005;162:2302-2308.

7. Rehner TA, Kolbo JR, Trump R, Smith C, Reid D. Depression among victims of south Mississippi's methyl parathion disaster. Health Soc Work. 2000;25:33-40

8. Taylor S, Asmundson GJ, Carleton RN, Brundin P. Acute posttraumatic stress symptoms and depression after exposure to the 2005 Saskatchewan Centennial Air Show disaster: prevalence and predictors. Am J Disaster Med. 2007;2:217-230.

9. Chou FH, Su TT, Chou P, Ou-Yang WC, Lu MK, Chien IC. Survey of psychiatric disorders in a Taiwanese village population six months after a major earthquake. J Formos Med Assoc. 2005;104:308-317.

10. Seplaki CL, Goldman N, Weinstein M, Lin YH. Before and after the 1999 Chi-Chi earthquake: traumatic events and depressive symptoms in an older population. So Sci Med. 2006;62:3121-3132.

11. Grieger TA, Cozza SJ, Ursano RJ, Hoge C, Martinez PE, Engel CC, et al. Posttraumatic stress disorder and depression in battle-injured soldiers. Am J Psychiatry. 2006;163:1777-1783.

12. Heptinstall E, Sethna V, Taylor E. PTSD and depression in refugee children: associations with pre-migration trauma and post-migration stress. Eur Child Adolesc Psychiatry. 2004;13:373-380.

13. Kashdan TB, Morina N, Priebe S. Post-traumatic stress disorder, social anxiety disorder, and depression in survivors of the Kosovo War: experiential avoidance as a contributor to distress and quality of life. J Anxiety Disord. 2009;23:185-196.

14. Silove D, Steel Z, Susljik I, Frommer N, Loneragan C, Chey T, et al. The impact of the refugee decision on the trajectory of PTSD, anxiety, and depressive symptoms among asylum seekers: a longitudinal study. Am J Disaster Med. 2007;2:321-329.

15. Beard JR, Tracy M, Vlahov D, Galea S. Trajectory and socioeconomic predictors of depression in a prospective study of residents of New York City. Ann Epidemiol. 2008;18:235-243.

16. Bonanno GA. Loss, trauma, and human resilience: have we underestimated the human capacity to thrive after extremely aversive events? Am Psychol. 2004;59:20-28.

17. Nandi A, Beard JR, Galea S. Epidemiologic heterogeneity of common mood and anxiety disorders over the lifecourse in the general population: a systematic review. BMC Psychiatry. 2009;9:31. 
18. Jung T, Wickrama KAS. An introduction to latent class growth analysis and growth mixture modeling. Social and Personality Psychology Compass. 2008;2:302-317.

19. Nagin D, Tremblay RE. What has been learned from group-based trajectory modeling? Examples from physical aggression and other problem behaviors. Ann Am Acad Pol Soc Sci.. 2005;602:82-117.

20. Nagin D. Analyzing developmental trajectories: a semiparametric, groupbased approach. Psychol Methods. 1999;4:139-157.

21. Galea S, Vlahov D, Resnick H, Ahern J, Susser E, Gold J, et al. Trends of probable post-traumatic stress disorder in New York City after the September 11 terrorist attacks. Am J Epidemiol. 2003;158:514-524.

22. Spitzer RL. Structured clinical interview for DSM-III-R-non-patient version. New York: Biometrics Research Department, New York State Psychiatric Institute; 1987.

23. Diagnostic and statistical manual of mental disorders, fourth edition. Washington (DC): American Psychiatric Association; 1994.

24. Boscarino JA, Galea S, Ahern J, Resnick H, Vlahov D. Utilization of mental health services following the September 11th terrorist attacks in Manhattan, New York City. Int J Emerg Ment Health. 2002;4:143-155.

25. Derogatis LR. Brief symptom inventory 18 (BSI-18) manual. Minnetonka (MN): NCS Assessments. 2001.

26. Boscarino JA, Galea S, Adams RE, Ahern J, Resnick H, Vlahov D. Mental health service and medication use in New York City after the September 11, 2001, terrorist attack. Psychiatr Serv. 2004;55:274-283.

27. Galea S, Boscarino JA, Resnick H, Vlahov D. Mental health in New York City after the September 11 terrorist attacks: results from two population surveys. In: Manderscheid RW, Henderson MJ, eds. Mental health, United States, 2002. Washington (DC): Government Printing Office; 2003.

28. Sherbourne CD, Stewart AL. The MOS social support survey. Soc Sci Med. 1991;32:705-714.
29. Ahern J, Galea S, Fernandez WG, Koci B, Waldman R, Vlahov D. Gender, social support, and posttraumatic stress in postwar Kosovo. J Nerv Ment Dis. 2004;192:762-770.

30. Centers for Disease Control and Prevention. Diagnostic Interview Schedule (DIS). Health status of Vietnam veterans. Atlanta (GA): CDC; 1989 405-499.

31. Lawyer SR, Resnick HS, Galea S, Ahern J, Kilpatrick DG, Vlahov D. Predictors of peritraumatic reactions and PTSD following the September 11th terrorist attacks. Psychiatry. 2006;69:130-141.

32. Bureau of the Census. Census summary tape (STF3A). Washington (DC): 2000.

33. Jones BL, Nagin D, Roeder K. A SAS procedure based on mixture models for estimating developmental trajectories. Sociol Methods Res. 2001;29:374-393.

34. Adams RE, Boscarino JA. Differences in mental health outcomes among Whites, African Americans, and Hispanics following a community disaster. Psychiatry. 2005;68:250-265.

35. Galea S, Vlahov D, Tracy M, Hoover DR, Resnick H, Kilpatrick D. Hispanic ethnicity and post-traumatic stress disorder after a disaster: evidence from a general population survey after September 11, 2001. Ann Epidemiol. 2004;14:520-531.

36. Sachs-Ericsson N, Plant EA, Blazer DG. Racial differences in the frequency of depressive symptoms among community dwelling elders: the role of socioeconomic factors. Aging Ment Health. 2005;9:201-209.

37. Lorant V, Croux C, Weich S, Deliege D, Mackenbach J, Ansseau M. Depression and socio-economic risk factors: 7-year longitudinal population study. Br J Psychiatry. 2007;190:293-298.

38. Paulsen AS, Crowe RR, Noyes R, Pfohl B. Reliability of the telephone interview in diagnosing anxiety disorders. Arch Gen Psychiatry. 1988;45:62-63.

39. Rohde P, Lewinsohn PM, Seeley JR. Comparability of telephone and faceto-face interviews in assessing axis I and II disorders. Am J Psychiatry. 1997;154:1593-1598. 